\title{
Protocolo de Roteamento de Veículos Fundamentado na Taxa de Ocupação da Via para Redes Veiculares
}

\author{
Thiago S. Gomides ${ }^{1}$ e Daniel L. Guidoni ${ }^{1}$ \\ ${ }^{1}$ Departamento de Ciência da Computação \\ Universidade Federal de São João Del Rei (UFSJ), MG - Brasil \\ thiagomides@gmail.com e guidoni@ufsj.edu.br
}

\begin{abstract}
The exponential growth in the number of vehicles has become a constant concern in the big cities. Annually, a billion-dollar deficit is observed due to fuel costs and vehicle maintenance. The impact of this chaos on the streets has been a constant topic of discussions among governments, researchers and environmentalists. With the purpose of reducing congestion, this work proposes a solution for the reorganization of vehicles based on the occupancy rate of the roads. During its trip, the vehicle notifies the congestion service it positions. Periodically, this service uses the occupation rate of the roads to find alternative routes. Simulation results shows that the proposed solution is able to reduce travel time with a small impact on the traveled distance.
\end{abstract}

Resumo. O crescimento exponencial do número de veículos tem se tornado uma constante preocupação nas grandes cidades. Anualmente, um deficit bilionário é observado devido à gastos com combustíveis e à manutenção de veículos. $O$ impacto desse caos nas ruas vem sendo pauta constante de discussões entre governos, pesquisadores e ambientalistas. Com o propósito de diminuir congestionamentos, este trabalho propõe uma solução para a reorganização dos veículos com base na taxa de ocupação das vias. Durante o seu percurso, o veículo notifica a sua posição para o serviço de congestionamento. Periodicamente, este serviço utiliza a taxa de ocupação das vias para encontrar rotas alternativas e com menor tempo de duração. Resultados de simulação indicam que a solução proposta é capaz de reduzir o tempo de viagem com um pequeno impacto na distância percorrida.

\section{Introdução}

O aumento do número de veículos tem se tornado um problema nos grandes centros urbanos. Economicamente, bilhões de reais são desperdiçados a cada ano devido aos gastos extras com combustíveis e manutenção, ocasionados pela lentidão no trânsito [Trentin 2015]. Além dos danos financeiros, e dos prejuízos ao meio ambiente, são percebidos efeitos à qualidade de vida dos motoristas e passageiros. Segundo o Instituto de Estudos Avançados da Universidade de São Paulo [Galvão 2013], somente na cidade de São Paulo gasta-se cerca de 200 milhões de litros de gasolina e álcool além de outros 4 milhões de litros de diesel por ano, devido à retenção severa do fluxo de veículos, ocorridos em sua maior parte em horários de movimento intenso.

Em contrapartida, com o objetivo de reduzir as agressões ao meio ambiente e o tempo em trânsito, surge a proposta de reorganizar os veículos para evitar a sobrecarga 
das vias. Esta técnica conhecida como roteamento de veículos, consiste na análise de um cenário congestionado e na proposta de decisões inteligentes baseadas em rotas alternativas [Barba et al. 2012]. Dentre as inúmeras formas de reorganização do tráfego, a utilização de Sistemas de Transportes Inteligentes (STI) [Bazzan and Klügl 2007] tem ganhado foco nos últimos anos, principalmente, pela constante incorporação de diferentes tecnologias de comunicação e processamento nos veículos automotores que, desta forma, contribuem para o surgimento de soluções direcionadas e eficientes.

Soluções inspiradas em STI podem ser implementadas por meio das redes veiculares (VANETs - Vehicular Ad-Hoc Networks). As VANETs são um conjunto de protocolos de comunicação móvel que visam proporcionar conforto e segurança aos usuários de automóveis e, sobretudo, uma interação amigável entre condutor e veículo. Este, equipado com GPS e comunicação sem fio é capaz de atuar como um sensor móvel e de obter informações sobre a localização, a velocidade e a rota - utilizada para o controle da mobilidade urbana. Para a redistribuição do fluxo dos automóveis, as redes veiculares têm por objetivo estabelecer métricas de detecção, quantificação e modelos funcionais de reorganização dos veículos, alguns destes em [Tonguz et al. 2010] e [Villas et al. 2014]. Em geral, as soluções visam a diminuição dos congestionamentos e a prevenção da perpetuação destes, principalmente em decorrência do desvio do fluxo das vias.

O objetivo deste trabalho é propor um algoritmo integrado para classificar e reorganizar o fluxo de veículos de modo a minimizar os congestionamentos. A solução proposta baseia-se na quantidade de automóveis presentes nas vias para a classificação do fluxo. A partir desta, cria-se um grafo direcionado para representar as trajetórias da cidade e, dessa forma, este é ponderado com base na quantidade de veículos em um mesmo segmento sendo definidas novas rotas para o deslocamento. A solução proposta foi avaliada e comparada com o protocolo de reorganização de veículos Dynamic Shortest Path (DSP) e apresenta melhores resultados em termos de tempos de viagem, de espera e da distância percorrida.

O restante deste artigo está organizado como segue. A Seção 2 apresenta os principais trabalhos relacionados. A Seção 3 detalha o funcionamento do protocolo proposto. As simulações e os resultados do estudo da avaliação e do desempenho do protocolo são apresentados na Seção 4. Por fim, a Seção 5 apresenta as conclusões e os potenciais trabalhos futuros.

\section{Trabalhos Relacionados}

O roteamento veicular é uma técnica destinada para ambientes em que o volume de automóveis é intenso, o deslocamento ocorre em baixa velocidade e há constantes interrupções do fluxo. Caso contrário, os veículos tendem a seguir os menores caminhos entre os pontos de partida e o de chegada. Em [Lomax 1997] os autores determinam que o surgimento de congestionamentos está relacionado ao tempo do percurso em regiões com a retenção intensa em comparação à duração em condições normais. Ademais, diversas são as soluções propostas para a reorganização do fluxo de veículos que utilizam as VANETs [de Souza and Villas 2015], [Brennand et al. 2015] e [Meneguette et al. 2016]. Em geral, as soluções a partir das estimativas obtidas no trânsito - para a detecção e reorganização de veículos - tem como objetivo reduzir e, simultaneamente, não realocar o congestionamento para as demais regiões da cidade. 
A metodologia proposta por [de Souza and Villas 2015] tem por objetivo evitar que as retenções no tráfego sejam formadas em decorrência de imprevistos, como acidentes. Para isso, a solução compartilha a informação do perigo por meio da comunicação entre veículos por múltiplos saltos. Esse protocolo tenta evitar a aproximação de novos veículos na região de intervenção e controlar o congestionamento causado pelos automóveis próximos ao acidente. A avaliação é realizada em comparação ao cenário ausente de roteamento e apresenta uma redução no consumo médio de $86 \%$.

A utilização de grafos em redes veiculares é apresentada por [Brennand et al. 2015]. Neste trabalho, é proposto um protocolo de comunicação entre o veículo e a infraestrutura auxiliar (RoadSide Unit), que aplica as estimativas de trânsito obtidas na construção de um grafo ponderado da rede. Nesse sentido, as RSU são necessárias para o funcionamento do protocolo, uma vez que, torna-se essencial a total cobertura de comunicação do cenário. As estimativas obtidas são encaminhadas em direção à central de processamento onde as decisões de roteamento são calculadas.

Em [Pan et al. 2017] os autores apresentam uma solução fundamentada nos tempos de deslocamentos das vias, chamado de Dynamic Shortest Path (DSP). A alternativa, de maneira similar ao trabalho proposto neste artigo, emprega um serviço centralizado para o cálculo de novas rotas. Dessa forma, sempre que um veículo passa por uma interseção, notifica ao serviço central sobre o tempo de viagem verificado na última via do percurso. Ademais, quando este aproxima-se de um congestionamento, o sistema central calcula novas rotas a partir dos tempos de viagens das vias. Sendo assim, devido à similaridade com a alternativa proposta neste artigo - solução global e centralizada, utiliza-se uma contraposição do protocolo exposto em detrimento daquela difundida na literatura (DSP) na seção de resultados.

\section{Solução Proposta}

Neste segmento, apresenta-se o protocolo que, por objetivo, visa a redução dos congestionamentos. Dessa maneira, este foi divido em duas partes: (i) classificação e (ii) reorganização dos veículos. Na fase (i), os automóveis notificam ao serviço de congestionamento sua localização na cidade (via). Além disso, fundamentado na densidade das vias, a fase (ii) é executada: o serviço verifica a existência de rotas descongestionadas para cada automóvel. Desse modo, a partir da existência de uma melhor rota, este é notificado e altera o seu percurso.

\subsection{Classificação de Congestionamento}

Além do mais, o trabalho consiste na avaliação das condições de um cenário urbano com tráfego intenso e na elaboração de rotas alternativas para os veículos, com o intuito de minimizar o tempo em trânsito. Para isso, é preciso mensurar e classificar o número de automóveis a partir da utilização das estimativas do deslocamento em cada região. Porquanto, existem diferentes formas na literatura para verificar as condições do trânsito, como: o encaminhamento de dados via redes móveis $3 \mathrm{G}$ ou $4 \mathrm{G}$, a comunicação entre veículos ao transmitir beacons [Meneguette et al. 2016], a instalação de sensores em semáforos [Barba et al. 2012, Rakha and Kamalanathsharma 2011], a utilização de RSU [Brennand et al. 2015] e o Sistema de Posicionamento Global (GPS). Neste trabalho, considera-se que cada veículo esteja equipado com um receptor GPS, possui o 


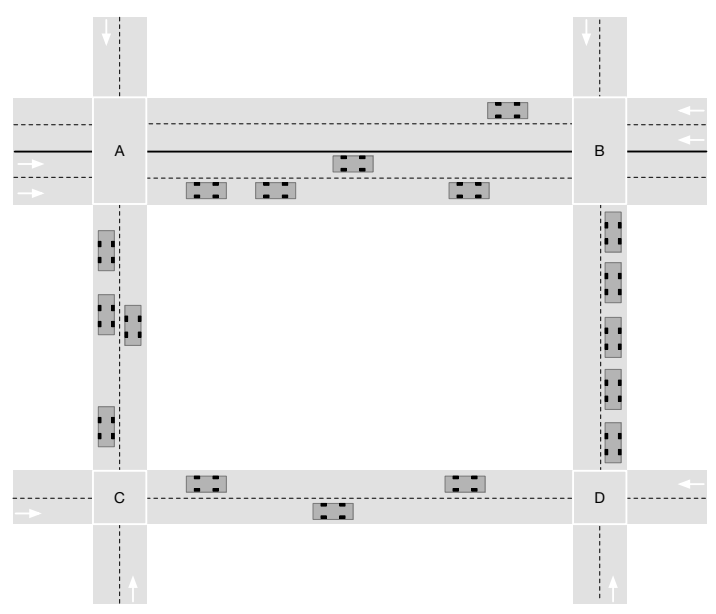

(a) Quantidade de veículos nas vias

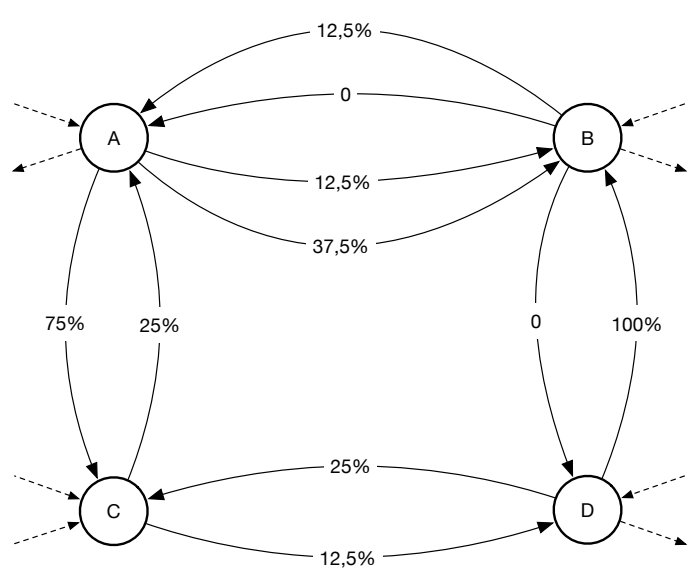

(b) Grafo com as respectivas taxas de ocupação

Figure 1. Representação das vias.

mapa digital (vias) da cidade e comunica-se com o serviço de reorganização de rotas por transmissão através do celular.

Outrossim, a Figura 1(a) ilustra a representação das vias na solução proposta. Além disso, as taxas de ocupação das respectivas ruas são apresentadas na Figura 1(b), as interseções destas (pontos A, B, C e D) são representadas como vértices e cada via sendo definida como uma aresta direcionada no grafo, onde o peso destas representa sua ocupação. Dessa maneira, sempre que um veículo alterna o seu segmento ocorre a notificação ao serviço de congestionamento para a atualização da taxa de ocupação. Além do mais, a mensagem contém o identificador da atual via e o destino do automóvel.

Ademais, classifica-se o congestionamento das vias com base na teoria de roteamento de pacotes da internet [L. Buriol and Ribeiro 2003], onde o fluxo de veículos é caracterizado em cinco níveis de acordo com a Taxa de Ocupação (TO) da rua: Livre quando $T O<30 \%$, Fluindo quando $30 \%<T O \leq 60 \%$, Lento quando $60 \%<T O \leq 90 \%$, Intenso quando $90 \%<T O<100 \%$ e por fim Caótico quando $T O=100 \%$.

\subsection{Reorganização dos Veículos}

Além disso, com o intuito de reduzir a retenção do tráfego urbano e, simultaneamente, a não realocação do fluxo para outra região da cidade, a solução proposta elabora novos caminhos de maneira centralizada e avalia a densidade das vias. Assim, busca-se uma solução global eficiente. Na proposta, utiliza-se o cálculo de rotas em ciclos de 1 minuto de duração - intervalo considerável para a percepção dos efeitos da reorganização dissertada no período anterior.

A cada ciclo, os veículos são avaliados e novas rotas podem ser estabelecidas. Dessa forma, para cada automóvel calcula-se o caminho mínimo entre a sua localização e o seu destino e, com isso, as arestas pertencentes ao novo percurso são atualizadas no grafo a partir da função de densidade representada na Figura 2 e onde a taxa de ocupação da via possui um impacto no peso das arestas. Desse modo, quanto mais congestionados estiverem os segmentos, maior será o seu índice de sobrecarga e menor será a chance de uma nova rota utilizá-los como destino. Com isso, a reorganização apenas estará final- 
izada quando não existirem mais veículos a serem analisados.

$$
f(x)=\left\{\begin{array}{rc}
1, & x \leq 0.3 \\
3, & 0.3<x \leq 0.6 \\
6, & 0.6<x \leq 0.9 \\
9, & 0.9<x<1 \\
10, & x=1 .
\end{array}\right.
$$

Figure 2. Cálculo da Porcentagem de Ocupação da Via

A reorganização dos automóveis pode ser feita de diferentes maneiras, tais como: de modo aleatório e com menor ou maior rota. O primeiro critério, compara os automóveis sem considerar o tamanho do seu deslocamento. No segundo critério, a lista dos veículos é ordenada de maneira crescente em relação ao tamanho da trajetória, sendo aquele com a menor rota o primeiro veículo a ser considerado. O terceiro critério avalia o automóvel de maior rota em detrimento daquele com a menor trajetória. Porquanto, as três foram analisadas e aquele de maior percurso obteve os melhores resultados. Ademais, este critério determina que os veículos com as trajetórias mais extensas sejam os primeiros a serem considerados e que percorram os caminhos com o menor fluxo, o que reduz o tempo das maiores viagens. Sendo assim, a solução proposta utiliza o terceiro critério para o roteamento dos veículos.

\section{Simulações e Resultados}

Esta seção descreve as ferramentas usadas, os cenários de simulação e os resultados obtidos. A princípio, a solução proposta foi comparada ao algoritmo da literatura chamado DSP e, também, ao modelo sem a reorganização do tráfego.

Outrossim, a mobilidade dos veículos foi simulada a partir da ferramenta SUMO $0.27 .1^{1}$. Porquanto, além de possuir código aberto, o SUMO é eficiente na simulação de grandes redes. Além do mais, para a manipulação e a construção da rede de veículos como um grafo foi utilizado a biblioteca networkx ${ }^{2}$. Com isso, as soluções foram avaliadas em detrimento do cenário representado por um trecho real do mapa da cidade de Los Angeles, EUA, sob uma região de $25 \mathrm{~km}^{2}$. Além disso, O trecho foi obtido e extraído do repositório da ferramenta OpenStreetMap ${ }^{3}$, tendo características similares a um Manhattan Grid, como pode ser observado na Figura 3.

Para avaliar os protocolos, foram aplicados os valores constantes de veículos no cenário: $1250,2500,3750,5000,6250$. Sendo, o primeiro aquele com os menores pontos de congestionamento e, o último, extremamente sobrecarregado. Assim sendo, cada veículo possui uma rota inicial (caminho mínimo) entre duas ruas aleatórias no mapa com no mínimo $1 \mathrm{~km}$ de extensão. Porquanto, todos os gráficos apresentados nesta seção foram obtidos a partir de uma distribuição T Student com média de 10 simulações e com o intervalo de confiança de $95 \%$. Além disso, as métricas avaliadas foram calculadas por meio da média entre os veículos do cenário.

\footnotetext{
${ }^{1}$ http://sumo.dlr.de/

${ }^{2} \mathrm{https}: / /$ networkx.github.io/

${ }^{3} \mathrm{https}: / /$ www.openstreetmap.org
} 


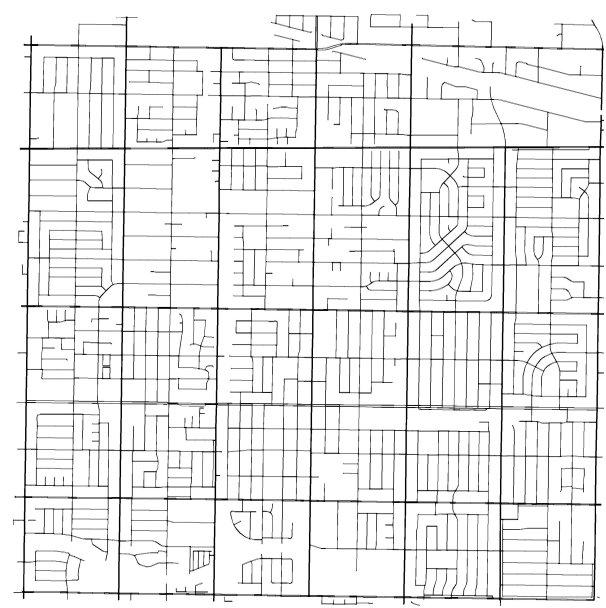

Figure 3. Fragmento de $25 \mathrm{~km}^{2}$ da malha rodoviária da cidade de Los Angeles.

A Figura 4(a) ilustra o Tempo de Duração das viagens para diferentes quantidades de veículo. Sendo, o tempo de percurso, a média da duração dos deslocamentos destes. Além do mais, quando a rede possui poucos pontos de congestionamento (1250 veículos), as três soluções possuem tempos de viagens similares. Ademais, para as redes com 3250 automóveis, o modelo proposto apresenta duração média $21 \%$ e $39 \%$ inferior em comparação às soluções DSP e Original, respectivamente. Também, quando a rede possui muitos carros (6250 veículos) e pontos de congestionamento, a proposta reduz em $42 \%$ o período de viagem em comparação ao DSP e em $57 \%$ à solução sem a reorganização dos veículos.

O Tempo Perdido apresenta as interrupções durante o trajeto, considera o tempo em repouso, as eventuais desacelerações e encontra-se ilustrado na Figura 4(b). Porquanto, mesmo com uma pequena quantidade de veículos, a solução proposta apresenta um ganho significativo em comparação aos demais protocolos. Além disso, quando a rede possui 1250 veículos, o modelo apresentado reduz em 55 e 33\% o tempo em relação à solução Original e DSP, respectivamente. Por fim, quando a rede possui sobrecarga veicular (6250 automóveis), o protocolo é capaz de minimizar em 44 e $72 \%$ o tempo em comparação ao DSP e à Original.

Outrossim, a Velocidade Média é apresentada na Figura 4(d) sendo o seu cálculo efetivamente significativo ao trânsito das grandes cidades. Sendo assim, torna-se importante o entendimento a respeito da relação entre esta e os congestionamentos. Dessa forma, o alto fluxo de veículos faz com que o deslocamento seja mais lento, o que aumenta o tempo de viagem, o tempo parado e o consumo de combustível. Ademais, ao avaliar a solução Original, encontra-se a menor velocidade média - consequência da alta quantidade de engarrafamento. Logo, esse modelo possui uma velocidade média $61 \%$ menor em relação à solução proposta e ao DSP, que possuem resultados similares. Além disso, diferentemente do DSP, o padrão exposto é capaz de aumentar a velocidade média ao utilizar apenas a taxa de ocupação da via.

A Distância Percorrida é apresentada na Figura 4(c). É importante observar que, na solução Original, os carros percorrem as menores rotas e, por isso, esta possui o menor deslocamento médio percorrido. As estratégias para reorganização alteram o 


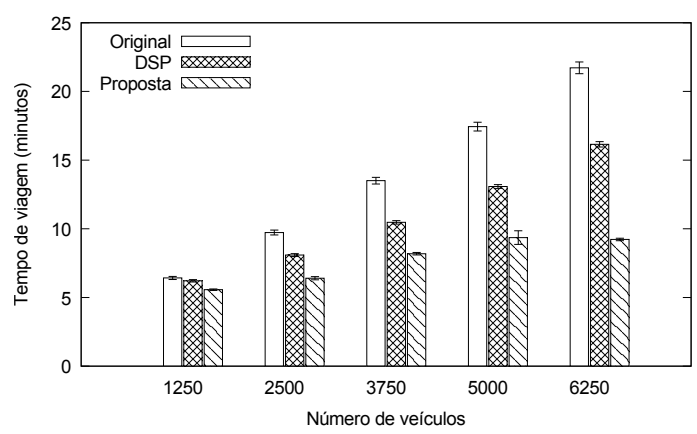

(a) Duração

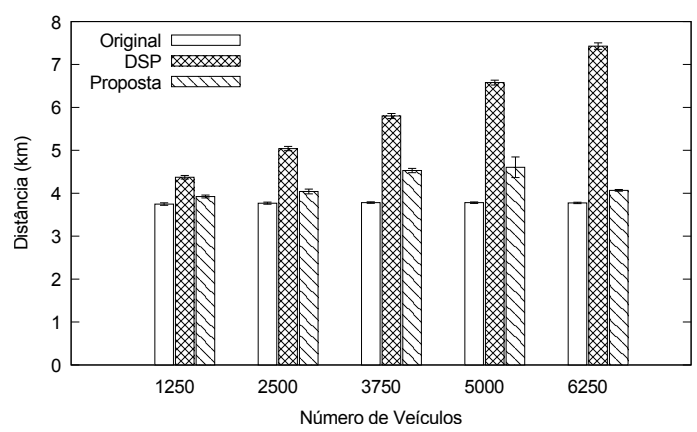

(c) Distância Percorrida

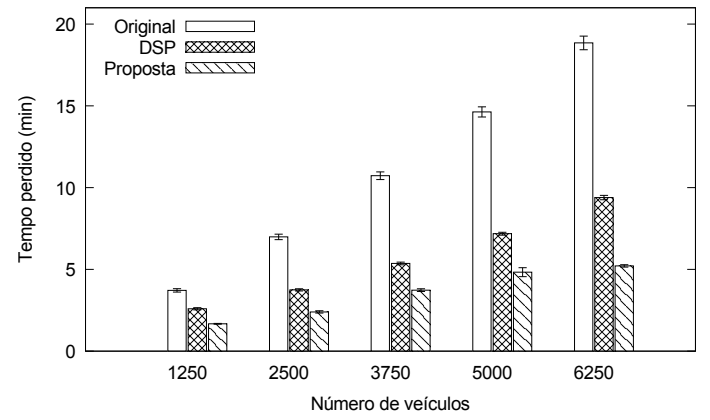

(b) Tempo Perdido

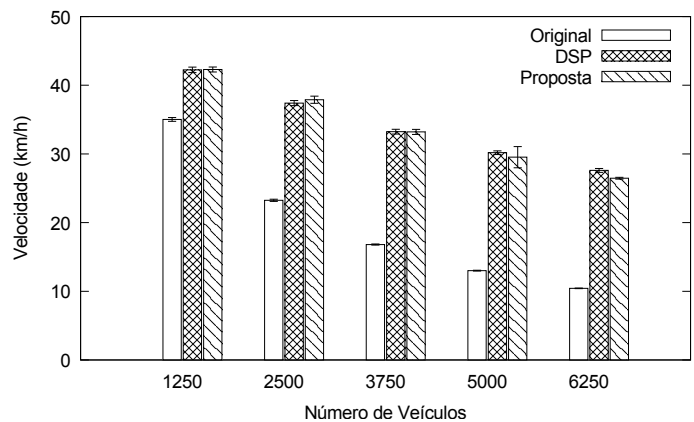

(d) Velocidade Média

Figure 4. Gráficos referentes a análises obtidas na execução do protocolo.

fluxo de vias e de regiões congestionadas para reduzir o tempo de viagem, entretanto, aumenta a distância percorrida. Além do mais, observar-se que o DSP eleva a trajetória a partir do aumento do fluxo de carros. No entanto, o proposto mantém um fator linear ao aumento da distância. Porquanto, quando a rede possui 3750 veículos, o DSP amplia a distância percorrida em $34 \%$ comparado à solução Original e $21 \%$ à proposta. Esta, aumenta em 16\% o deslocamento em comparação àquela sem roteamento. Dessa forma, percebe-se que a utilização da densidade das vias na reorganização dos veículos possui baixo impacto na distância percorrida e, ao mesmo tempo, reduz o tempo de viagem e aumenta a velocidade média. Ademais, o DSP consegue diminuir a duração do trajeto, mas eleva consideravelmente o percurso.

\section{Conclusões}

Este trabalho apresenta uma solução para a realocação das rotas dos automóveis em cenários de congestionamento urbano. $\mathrm{O}$ modelo proposto realiza a classificação do alto fluxo e, com base na taxa de ocupação das vias, sugere novas rotas para os veículos. Ademais, a proposta possui como objetivo reduzir e, simultaneamente, não desviar o congestionamento para outra região da cidade. Dessa forma, os resultados das simulações demonstram o desempenho da nossa solução que, quando comparada à solução Original e ao DSP, diminui o tempo do trajeto e o de espera aumentando a velocidade média com pouco impacto na distância percorrida.

Nesse sentido, como trabalhos futuros, pretende-se a descentralização do processamento por meio de mecanismos de análise distribuída sem que, para isso, os ganhos sejam minimizados. Por fim, consolidar a avaliação do desempenho em cenários maiores 
e reais.

\section{References}

Barba, C. T., Mateos, M. A., Soto, P. R., Mezher, A. M., and Igartua, M. A. (2012). Smart city for vanets using warning messages, traffic statistics and intelligent traffic lights. In 2012 IEEE Intelligent Vehicles Symposium, pages 902-907.

Bazzan, A. L. and Klügl, F. (2007). Sistemas inteligentes de transporte e tráfego: uma abordagem de tecnologia da informação. Anais das Jornadas de Atualização em Informática, pages 2296-2337.

Brennand, C. A. R. L., de Souza, A. M., Maia, G., Boukerche, A., Ramos, H., Loureiro, A. A. F., and Villas, L. A. (2015). An intelligent transportation system for detection and control of congested roads in urban centers. In 2015 IEEE Symposium on Computers and Communication (ISCC), pages 663-668.

de Souza, A. M. and Villas, L. A. (2015). A new solution based on inter-vehicle communication to reduce traffic jam in highway environment. IEEE Latin America Transactions, 13(3):721-726.

Galvão, A. (2013). O impacto do caos nas ruas. http://veja.abril.com.br/ idade/exclusivo/transito/contextol.html.

L. Buriol, P. França, M. R. and Ribeiro, C. (2003). Otimizando o roteamento do tráfego na internet. In Anais do XXXV SBPO, pages 1722-1732.

Lomax, T, T. S. S. G. L. H. P. R. B. P. . D. (1997). Quantifying Congestion: Volume 1, Final Report (NCHRP Report 398). Transportation Research Board.

Meneguette, R., Fillho, G., Bittencourt, L., Ueyama, J., and Villas, L. (2016). A solution for detection and control for congested roads using vehicular networks. IEEE Latin America Transactions, 14(4):1849-1855.

Pan, J., Popa, I. S., and Borcea, C. (2017). Divert: A distributed vehicular traffic rerouting system for congestion avoidance. IEEE Transactions on Mobile Computing, 16(1):58-72.

Rakha, H. and Kamalanathsharma, R. K. (2011). Eco-driving at signalized intersections using v2i communication. In 2011 14th International IEEE Conference on Intelligent Transportation Systems (ITSC), pages 341-346.

Tonguz, O. K., Wisitpongphan, N., and Bai, F. (2010). Dv-cast: A distributed vehicular broadcast protocol for vehicular ad hoc networks. IEEE Wireless Communications, 17(2):47-57.

Trentin, C. (2015). Trânsito de são paulo gera prejuízo de r\$ 80 bilhões ao ano. https://www.ecommercebrasil.com.br/artigos/ transito-de-sao-paulo-gera-prejuizo-de-r-80-bilhoes-ao-ano/.

Villas, L. A., Boukerche, A., Maia, G., Pazzi, R. W., and Loureiro, A. A. (2014). Drive: An efficient and robust data dissemination protocol for highway and urban vehicular ad hoc networks. Computer Networks, 75, Part A:381 - 394. 